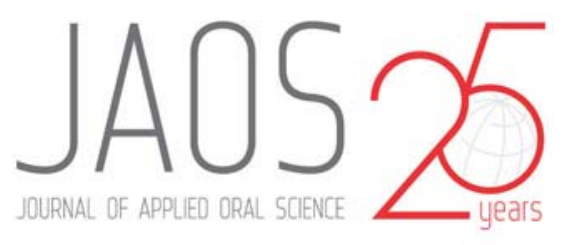

\title{
Metrical analysis of disc-condyle relation with different splint treatment positions in patients with TMJ disc displacement
}

\section{Abstract}

Mu-Qing LIU1,2,3

Jie LEI ${ }^{1,2,3}$

Jian-Hui HAN ${ }^{1,2,3}$

Adrian U-Jin $\mathrm{YAP}^{4,5,6}$

Kai-Yuan FU1,2,3
Submitted: September 20, 2016 Modified: December 2, 2016 Accepted: February 2, 2017 Center for TMD \& Orofacial Pain and Department of
Oral \& Maxillofacial Radiology.
Peking University School \& Hospital of Stomatology. No. 22 Zhong Guan Cun South Ave. Beijing 100081 - P.R. China.

Phone: 86-10-82195342 - Fax: 86-10-62173402 e-mail: kqkyfu@bjmu.edu.cn
Objective: To evaluate the effect of bite positions characterizing different splint treatments (anterior repositioning and stabilization splints) on the disc-condyle relation in patients with TMJ disc displacement with reduction (DDwR), using magnetic resonance imaging (MRI). Material and Methods: 37 patients, with a mean age of $18.8 \pm 4.3$ years ( 7 male and 30 females) and diagnosed with DDwR based on the RDC/TMD, were recruited. MRI metrical analysis of the spatial changes of the disc/condyle, as well as their relationships, was done in three positions: maximum intercuspation (Position 1 ), anterior repositioning splint position (Position 2), and stabilization splint position (Position 3). Disc/condyle coordinate measurements and disc condyle angles were determined and compared. Results: In Position 1, the average disc-condyle angle was $53.4^{\circ}$ in the 60 joints with DDwR, while it was $-13.3^{\circ}$ with Position 2 and $30.1^{\circ}$ with Position 3. The frequency of successful "disc recapture" with Position 2 was significantly higher (58/60, 96.7\%) than Position $3(20 / 60,33.3 \%)$. In Positions 2 and 3, the condyle moved forward and downward while the disc moved backward. The movements were, however, more remarkable with Position 2. Conclusions: Anterior repositioning of the mandible improves the spatial relationship between the disc and condyle in patients with DDwR. In addition to anterior and inferior movement of the condyle, transitory posterior movement of the disc also occurred.

Keywords: Temporomandibular joint. Mandibular condyle. Temporomandibular joint disc. Magnetic resonance imaging.

${ }^{1}$ Peking University School \& Hospital of Stomatology, Center for TMD \& Orofacial Pain and Department of Oral \& Maxillofacial Radiology, Beijing, China.

${ }^{2}$ National Engineering Laboratory for Digital and Material Technology of Stomatology, Beijing, China.

${ }^{3}$ Beijing Key Laboratory of Digital Stomatology, Beijing, China.

${ }^{4} \mathrm{Ng}$ Teng Fong General Hospital, Department of Dentistry, Jurong Health Services, Singapore, Singapore.

${ }^{5}$ SIM University, School of Science and Technology, Singapore, Singapore.

${ }^{6}$ National University of Singapore, Faculty of Dentistry, Singapore, Singapore. 


\section{Introduction}

Temporomandibular joint (TMJ) disc displacement is the most common type of TMJ arthropathy and involves an abnormal relationship or misalignment of the articular disc relative to the condyle. The usual direction for displacement of the disc is anteriorly or anterior-medially ${ }^{29}$. In spite of their apparent efficacy and widespread use for treating TMD, the precise mechanisms of action of oral splints remain controversial ${ }^{10}$. Hypotheses proposed include repositioning of condyle and/or articular disc, reduction in masticatory muscle activity, modification of patient's parafunctional behaviours, and changes in patient's occlusion $^{6}$. Two common types of oral splints used in clinical practice are the stabilization and anterior repositioning splints.

Anterior repositioning splints (ARS) have been shown to be effective for the management of disccondyle disorders ${ }^{14,18,26}$. Also known as anterior positioning appliances or mandibular orthopedic repositioning appliances, they serve to direct the mandibular condyle anteriorly in the glenoid fossa (i.e., protrusive mandibular position). The purpose of ARS treatment is not to alter the condylar position permanently, but to change it during the treatment to help the adaption of the retrodiscal tissues ${ }^{24}$. Based on clinical and MRI assessments, approximately $70 \%$ of reducing displaced discs was captured with the use of ARS $^{18}$. ARS could also alter mechanical stresses on the TMJ arising from the immediate physiologic improvement in the disc-condyle relationship ${ }^{4}$ and has been shown to facilitate regenerative remodeling of the TMJ ${ }^{22}$. Although the recaptured discs can occasionally move backward with successful ARS therapy, the amount of disc movement was found to be negligible ${ }^{19}$. The improved condyle-disc relationship with ARS was thought to be achieved primarily by the anteroinferior movement of the condyle.

Stabilization splints (SS) cover all the maxillary and mandibular teeth and are used to treat both joint and masticatory muscle disorders ${ }^{2,17}$. In contrast to ARS, SS are permissive appliances (allows for teeth to glide unimpeded over the biting surface) and do not protrude the mandible. They serve to provide a temporary and removable ideal occlusion at increased vertical dimension and centric relation. The use of SS increases TMJ space ${ }^{12}$ and allows for anteroinferior movement of the condyles ${ }^{7,11,16}$. SS are also used to manage disc-condyle disorders ${ }^{3}$. They are effective in eliminating the signs/symptoms of TMD, except TMJ clicking ${ }^{28}$. When compared to ARS for the treatment of TMJ DDwR, reduction in dysfunction and TMJ symptoms were found to be lower with SS therapy $5,25,27$.

The mechanism of action of both ARS and SS remains largely hypothetical. The two oral splints with their variance in bite and mandibular positioning can produce different degrees of disc and condyle positional changes, which in turn can affect treatment outcome. Most previous MRI studies were conducted on a single splint design with the between-subject method. Thus far, few studies have compared the two splint designs using a within-subject approach (every single participant is subjected to every single splint design) and at standardized vertical dimension. This study aimed to evaluate the effects of bite positions characterizing ARS and SS therapy (with and without anterior movement) on disc/condyle locations and disc-condyle relations in patients with TMJ DDwR, using MRI metrical analysis.

\section{Material and Methods}

\section{Patients}

A total of 37 patients, with a mean age of $18.8 \pm 4.3$ years (ranging from 12 to 30 years, 7 male and 30 female) and with complaints of TMJ clicking and/or intermittent locking, were recruited. All patients had permanent dentition, free of TMD-related pain, and 16 were younger than 18 years of age. To lessen the effect of confounding variables including marked skeletal/ occlusal discrepancies and systematic diseases, exclusion criteria were as follows: Presence of (1) crossbites and open bites; (2) deep overbites (vertical overlap of the maxillary central incisors $>1 / 2$ of the mandibular central incisors); (3) large overjets (>5 $\mathrm{mm})$; (4) rheumatic or degenerative joint diseases. The study was approved by the Biomedical Institutional Review Board. Written inform consent was obtained from all participating subjects.

Fifty-one (out of 74) joints of the 37 patients were clinically diagnosed with DDwR using the RDC/TMD ${ }^{9}$. Bilateral DDwR was observed in 14 patients. Upon MRI examination, 9 of the 23 clinically asymptomatic joints were also diagnosed with DDwR, based on the criteria defined by Ahmad, et al. ${ }^{1}$ (2009). The 51 joints 
with clinical diagnosis of DDwR were all confirmed by MRI examination. Thus, a total of 60 joints with DDwR were included in this study. 4 asymptomatic joints were diagnosed with DDwoR and 10 joints were found to be normal with both clinical and MRI assessment.

\section{Determination of bite and mandibular positions}

The condyle and disc locations were assessed in three bite positions: Position 1: maximal intercuspation (MICP); Position 2: characterizing ARS position; Position 3: characterizing SS position (Figure 1). For Position 1, subjects were asked to bite their back teeth completely together. The distance between the gingival margins of the left upper and lower central incisors (D1) and the overjet of the left upper central incisor were recorded using a caliper (Guanglu SF2000, Guangxi, China). For Position 2, subjects were asked to open their mouths fully beyond the clicking point and instructed to close in a protruded position with the incisors in an edge-to-edge relation. The mandibular position was registered using a silicone bite registration material (Occlufast Rock, Zhermack, Rovigo, Italy). The distance between the gingival margins of the left upper and lower central incisors (D2) was determined. For Position 3, subjects were asked to open fully beyond the clicking point and guided into the most retruded/rearmost mandibular locus. This was repeated several times till a reproducible "centric" relation position was achieved at the distance D2 without protrusion and registered. The distance between the gingival margins of the left upper and lower central incisors (D3) and the overjet of the left upper central incisor were again recorded. All bite registrations and mandibular measurements were made by a single investigator.

\section{Magnetic Resonance I maging}

MRI was performed with a 1.5-Tesla MR scanner (NOVUS, Siemens, Munich, Germany) with TMJ surface coils. Subjects were placed supine with their heads positioned with the Frankfurt plane perpendicular to the floor. The center beam was then lined up with the sagittal plane. All joints were scanned in the three mandibular positions in single visit using a factorial design order. For Positions 2 and 3, subjects were directed to open their mouths fully beyond the clicking point and gently close/bite into the prepared bite registrations. An initial low-resolution T1-weighted (TR $300 \mathrm{~ms}$; TE $10 \mathrm{~ms}$ ) axial localizing scan was followed by Proton-weighted (TR 1760 ms, TE 15 ms) oblique sagittal scan acquired vertical to the long axis of each condyle. The field of view was $12 \times 12 \mathrm{~cm}$ and matrix size was $512 \times 512$. Slice thickness and inter-slice spacing were set at $2 \mathrm{~mm}$ and $1 \mathrm{~mm}$, respectively.

\section{Metrical and statistical analysis}

The images were analyzed using image analysis software (Volview 3.4, Kitware, New York). The slice through the center of the horizontal long axis of the condyle was selected for evaluation (Figure 1). The disc-condyle angle was determined according to the method described by Drace and Enzmann ${ }^{8}$ (1990) (Figure $2 \mathrm{~A}$ ). The normal range for disc-condyle angle is between $-15^{\circ}$ to $15^{\circ 1}$. J oints with disc-condyle angles greater than $15^{\circ}$ are considered to have anterior disc displacement.

$X-Y$ coordinate measurements were used to determine disc and condyle positions (Figure 2B). The condyle and disc positions were expressed as $C$ and $D$ point coordinates $(x, y)$, respectively. The MRI data were evaluated by a trained radiologist who was blinded to clinical information. Intra-class correlation coefficients (ICC) were used to determine the intraand inter-observer reliability. A mean intra-observer ICC of $>0.900$ was established for all variables (the radiologist made all measurements twice with a one-week interval). Inter-observer ICC ranged from 0.868 to 1 for the different variables. The latter was determined with the assistance of another independent radiologist who was also blinded to patients' clinical data.

Disc and condyle $X-Y$ coordinates and disc-condyle angles for Positions 1, 2, and 3 were compared. SPSS version 20 (SPSS IBM, Chicago, USA) was used for statistical analysis. Data was subjected to one-way ANOVA (LSD) and t-test at significance level $P$ values $<0.05$.

\section{Results}

\section{Mandibular position}

The average vertical distance between the gingival margins of left upper and lower central incisors was $14.2 \pm 2.0 \mathrm{~mm}$ in Position 1 and $16.9 \pm 2.0 \mathrm{~mm}$ in Positions 2 and 3 . The average overjet of the left upper central incisor was $3.1 \pm 1.1 \mathrm{~mm}$ in Position 1 and $3.0 \pm 1.2 \mathrm{~mm}$ in Position 3. Position 2 thus postured the mandible downward and forward, while Position 

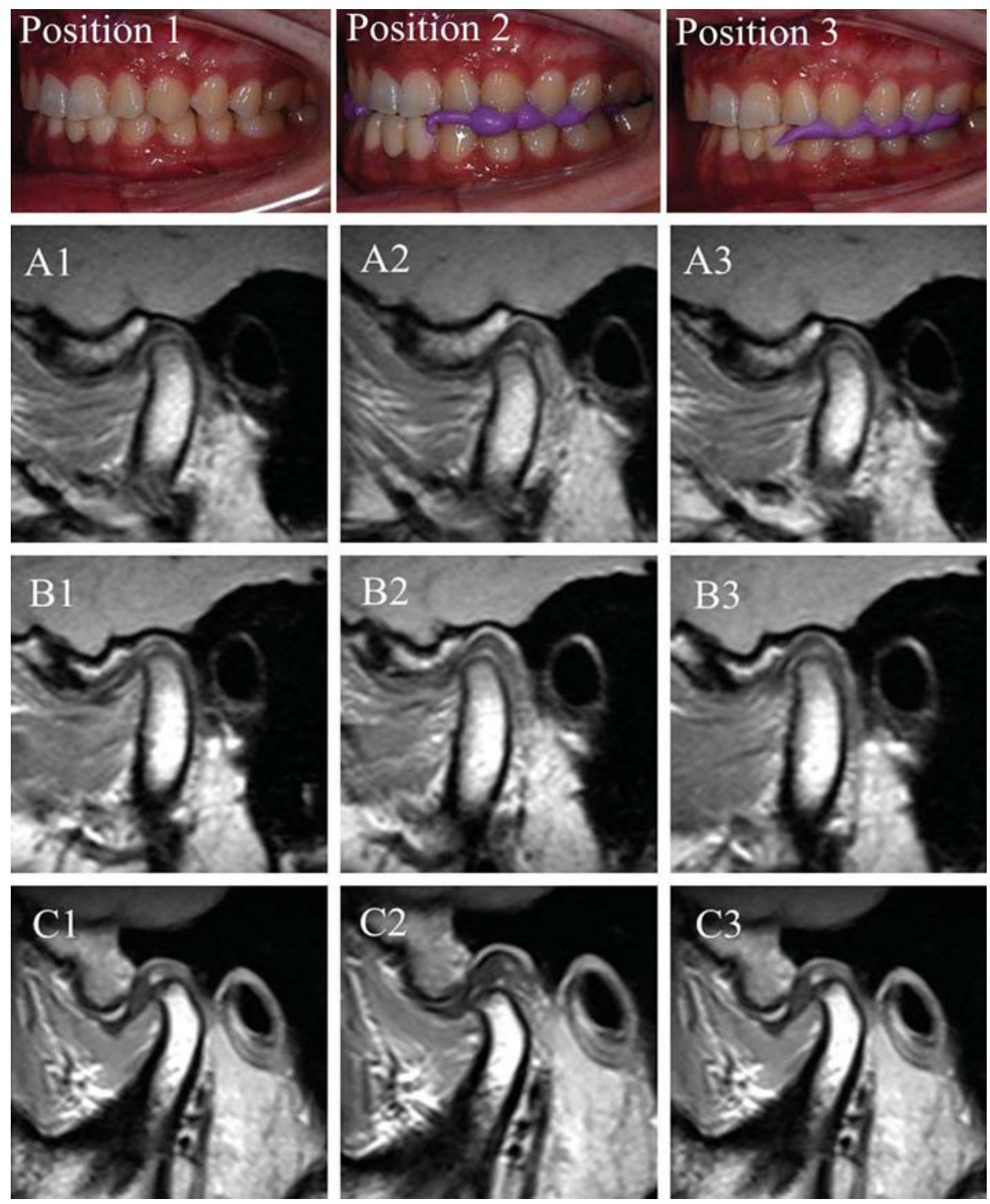

Figure 1- Representative MRIs of three joints in Position 1 (ICP - column 1), Position 2 (ARS - column 2), and Position 3 (SS - column 3). Joint $A$ was normal while Joints $B$ and $C$ were diagnosed with DDwR

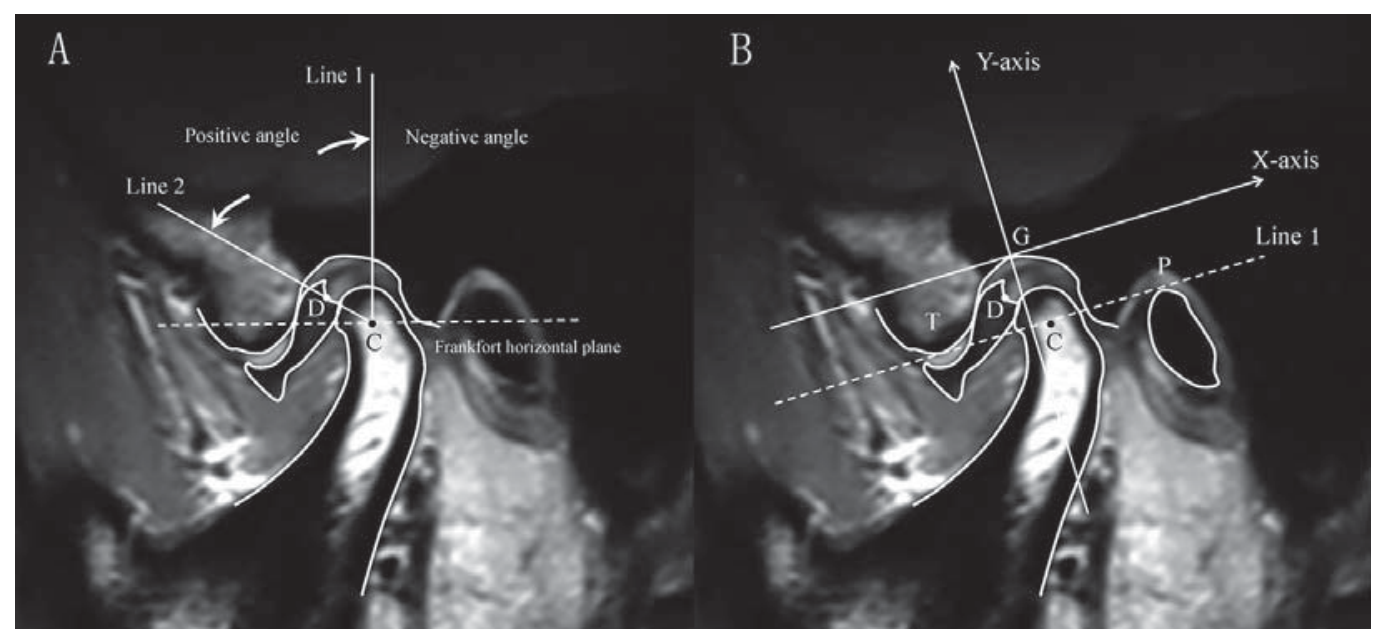

Figure 2- A: Drace's measurement for disc-condyle angle. $C$ point estimated the center of the condylar head. Line 1 was drawn from $\mathrm{C}$ point perpendicular to the Frankfort horizontal plane. Line 2 was drawn through $\mathrm{C}$ point to the midpoint of the posterior margin of the posterior band of the disc ( $\mathrm{D}$ point). The angle between line 1 and line 2 was defined as the disc-condyle angle. B: Coordinate measurement for disk and condyle position. A tangent from the lowest part of the articular tubercle $(T)$ to the highest edge of the porus acusticus externus $(P)$ was drawn (Line 1). The $X$-axis was drawn through the highest point of glenoid fossa $(G)$, parallel to Line 1. The $\mathrm{Y}$-axis was drawn from $\mathrm{G}$ point perpendicular to the $\mathrm{X}$-axis. $\mathrm{G}$ point was taken as the origin of coordinates 
Table 1- Disc-condyle angles for the three positions in normal and DDwR joints $\left({ }^{\circ}\right.$, mean $\left.\pm S D\right)$

\begin{tabular}{ccc}
\hline Positions & Normal joints $(\mathbf{n = 1 0})$ & DDwR joints $(\mathbf{n = 6 0 )}$ \\
\hline Position 1 & $-1.1 \pm 10.8^{\mathrm{a}}$ & $53.4 \pm 16.7^{\mathrm{a}}$ \\
Position 2 & $-11.7 \pm 12.0$ & $-10.5 \pm 17.1^{\mathrm{A}}$ \\
Position 3 & $-2.7 \pm 15.5^{\mathrm{a}}$ & $30.1 \pm 26.9^{\mathrm{a}, \mathrm{A}}$ \\
\hline
\end{tabular}

Lowercase letters in the same row indicate significant difference between two groups $(p<0.001)$

Uppercase letters in the same column indicate significant difference between two positions $(p<0.01)$

Table 2- Condyle and disc coordinates in normal and DDwR joints for Position 1, Position 2, and Position 3

\begin{tabular}{|c|c|c|c|c|c|}
\hline \multirow[t]{2}{*}{ Coordinates } & & \multirow{2}{*}{$\begin{array}{c}\text { Condyle } \\
\text { Normal joints } \\
(\mathrm{mm}, \text { mean } \pm S D)\end{array}$} & \multicolumn{3}{|c|}{ Disc } \\
\hline & & & $\begin{array}{c}\text { DDwR joints } \\
(\mathrm{mm}, \text { mean } \pm S D)\end{array}$ & $\begin{array}{c}\text { Normal joints } \\
(\mathrm{mm}, \text { mean } \pm S D)\end{array}$ & $\begin{array}{c}\text { DDwR joints } \\
(\mathrm{mm}, \text { mean } \pm S D)\end{array}$ \\
\hline \multirow[t]{3}{*}{ X coordinate } & Position 1 & $0.11 \pm 1.40^{\mathrm{a}, \mathrm{A}}$ & $1.21 \pm 1.19^{a, A}$ & $1.21 \pm 0.61^{\mathrm{b}, \mathrm{A}}$ & $-2.02 \pm 1.50^{\mathrm{b}, \mathrm{A}}$ \\
\hline & Position 2 & $-2.11 \pm 2.14^{\mathrm{A}, \mathrm{B}}$ & $-2.07 \pm 1.53^{\mathrm{A}}$ & $0.28 \pm 1.07^{A}$ & $0.21 \pm 1.67^{A}$ \\
\hline & Position 3 & $-0.59 \pm 1.27^{\mathrm{B}}$ & $0.24 \pm 1.32^{A}$ & $0.71 \pm 0.77^{a}$ & $-1.27 \pm 1.92^{\mathrm{a}, \mathrm{A}}$ \\
\hline \multirow[t]{3}{*}{ Y coordinate } & Position 1 & $-7.02 \pm 0.80^{\mathrm{a}, \mathrm{C}}$ & $-6.36 \pm 0.94^{\mathrm{a}, \mathrm{B}}$ & $-1.70 \pm 0.43^{\mathrm{b}, \mathrm{B}}$ & $-2.81 \pm 1.05^{b}$ \\
\hline & Position 2 & $-8.51 \pm 0.82^{\mathrm{C}, \mathrm{D}}$ & $-8.96 \pm 1.10^{\mathrm{B}}$ & $-2.34 \pm 0.41^{\mathrm{a}, \mathrm{B}, \mathrm{C}}$ & $-2.96 \pm 0.81^{a}$ \\
\hline & Position 3 & $-7.08 \pm 0.87^{D}$ & $-7.04 \pm 1.11^{\mathrm{B}}$ & $-1.66 \pm 0.42^{\mathrm{a}, \mathrm{C}}$ & $-2.76 \pm 0.92^{\mathrm{a}}$ \\
\hline
\end{tabular}

Lowercase letters in the same row indicate significant difference between two groups $(p<0.05)$

Uppercase letters in the same column indicate significant difference between two positions $(p<0.05)$

3 moved the mandible only downward with reference to Position 1.

\section{Disc-condyle angle}

Disc-condyle angle in normal and DDwR joints for Positions 1 to 3 are shown in Table 1 . In normal joints $(n=10)$, no significant difference in disc-condyle angle was observed between the three positions $(P>0.05)$. Disc-condyle angle was within the normal range $\left(-15^{\circ} \sim+15^{\circ}\right)$. In joints with DDwR $(n=60)$, mean disc-condyle angle was reduced from $53.4^{\circ}$ in Position 1 to $-10.5^{\circ}$ in Position 2 and $30.1^{\circ}$ in Position 3. The percentage of displaced disc reduction or disc "recapture" (post-treatment angle between $-15^{\circ}$ and $+15^{\circ}$ ) in DDwR joints was $96.7 \%$ (58/60 joints) for Position 2 and 33.3\% (20/60 joints) for Position 3 $(P<0.001)$. The average disc-condyle angle of DDwoR joints $(n=4)$ in Positions 1,2 , and 3 were $82.1^{\circ}, 65.5^{\circ}$, and $70.1^{\circ}$, respectively. No significant difference in disc-condyle angle was observed between the three positions $(P>0.05)$.

\section{Coordinate measurements of condyle and disc}

$\mathrm{C}$ points representing condylar positions in $\mathrm{X}$ - and Y-axis are shown in Table 2. C point movements were indicated by the numerical difference of coordinate values between two points. In normal joints, the condyle moved $2.22 \mathrm{~mm}$ forward and 1.49 downward from Position 1 to Position 2, and shifted $0.7 \mathrm{~mm}$ forward and $0.06 \mathrm{~mm}$ downward from Position 1 to 3. In joints with DDwR, the condyle moved 3.28 $\mathrm{mm}$ forward and $2.6 \mathrm{~mm}$ downward from Position 1 to 2 , and shifted $0.97 \mathrm{~mm}$ forward and $0.68 \mathrm{~mm}$ downward from Position 1 to Position 3. Statistical analysis indicated a significantly greater forward and downward movement of the condyle with ARS position when compared to the SS position.

$D$ points (posterior margin of the posterior band of disc) representing disc positions in the $\mathrm{X}$ - and $\mathrm{Y}$-axis are also presented in Table 2 . In normal joints, the $D$ point was located $1.21 \mathrm{~mm}$ behind and $1.70 \mathrm{~mm}$ below the $\mathrm{G}$ point (the highest point of glenoid fossa). The disc moved $0.93 \mathrm{~mm}$ forward and $0.64 \mathrm{~mm}$ downward from Position 1 to Position 2, while the condyle moved forward and downward. The disc did not move significantly from Position 1 to Position 3 in normal joints. In DDwR joints, the disc was located $2.02 \mathrm{~mm}$ anterior and $2.81 \mathrm{~mm}$ below the $\mathrm{G}$ point in Position 1 , indicating it was significantly displaced anteriorly and inferiorly when compared to normal joints (Table 2). In contrast to normal joints, the disc moved $2.23 \mathrm{~mm}$ backward from Positions 1 to 2 in DDwR joints. Disc movement from Position 1 to 3 was, however, only $0.75 \mathrm{~mm}$ backward. The coordinate values of $D$ point in both $\mathrm{X}$ - and $\mathrm{Y}$-axis for Position 2 in DDwR joints were similar to normal joints, indicating that the disc was fully reduced in the protrusive position. For all the measurements, no significant difference was detected 
between adolescent and adult patients.

\section{Discussion}

In this study, we determined the spatial changes in disc and condyle positions and the disc-condyle relation with mandibular positions of ARS and SS therapy in adolescents and adults. Metrical analysis was done with MRI, as it is a non-invasive diagnostic method that enables both qualitative and quantitative evaluation of the structures within the joint, including the TMJ disc. It is also generally painless and does not involve the use of ionizing radiation. As splint thickness can affect disc and condyle positions, a similar vertical dimension was maintained for both ARS and SS positions in this study. The use of mandibular positions mimicking ARS and SS instead of actual appliances negated the confounding effects of technical and clinical discrepancies associated with splint fabrication, adjustment, and use. The measurement method of disc-condyle angle and positions of condyle/disc was reliable and reproducible.

In the maximal intercuspation (Position 1), the disc in DDwR joints was displaced anteriorly and inferiorly, while the condyle was positioned backward and upward, in relation to normal joints. This corroborated the findings of earlier studies ${ }^{13,20}$. The condyle moved forward and downward in both ARS and SS treatment positions (Positions 2 and 3, respectively), but movement was more significant with ARS. The mechanism of action of ARS was previously thought to involve the "recapturing" of the discs, since the condyles are moved downward and forward. It was believed that the disc-condyle complex could be gradually walked back into the fossa by adjusting the biting surface of ARS $^{23}$. Kurita, et al. ${ }^{19}$ (1998) found that approximately $60 \%$ of the "recaptured" disc moved posteriorly, but the amount of movement was minor. In our study, disc movement was noticeably large (2.23 $\mathrm{mm}$ posteriorly) in joints with DDwR for the ARS position. The D point (posterior band of the disc) actually shifted back to the $G$ point (highest point of the glenoid fossa), indicating complete reduction of the displaced disc. In contrast, the displaced disc only moved back $0.75 \mathrm{~mm}$ for the SS position. The reduction of the displaced disc with ARS might be one of the key factors to the adaption and repair of the retrodiscal tissues. The elimination of joint clicking is commonly used to help determine the appropriate mandibular position for ARS ${ }^{19,26}$. In this study, the antero-inferior movement of the mandible for elimination of joint clicking is usually less than the protruded position with the incisors in an edge-to-edge relation. There may be a link between reduction of the displaced disc and the forward movement of the condyle. The stability of the reduced disc position, however, depends on maintaining the condyles in the forward and downward position, necessitating the fulltime use of ARS over a period of time. Upon stopping ARS use, reduced discs may once again get displaced, as the condyle moves posteriorly.

A proper disc-condyle-fossa relationship is thought to be important for normal TMJ function, alleviating joint pain, preventing degenerative joint changes, and promoting mandible growth in adolescents ${ }^{15,21}$. Although some joints with DDwR achieved normal disc-condyle relationship in the SS position (increased vertical without mandibular anterior positioning), the percentage of disc reduction was significantly lower than with ARS (33\% as opposed to $96.7 \%$ with ARS). Use of ARS achieved an immediate physiologic disccondyle-fossa relationship and increased the prospect of disc reduction. This explains in part their superior effectiveness in decreasing pain and dysfunction in patients with DDwR when compared to SS 5,25,27.

As with all studies, the current research has some limitations. Since actual oral splints were not fabricated, the influence of splint material stiffness on disc and condyle positions/relationships could not be ascertained. The long term effects of ARS and SS on disc-condyle-fossa relationships were also not determined. Patients with significant skeletal discrepancies, malocclusion, and rheumatic or degenerative joint diseases were excluded, which may also affect disc "recapture" in joints with DDwR.

\section{Conclusions}

In summary, anterior repositioning of the mandible improved the spatial relationship between the disc and condyle, increasing the prospect of disc reduction in patients with DDwR. In addition to anterior and inferior movement of the condyle, transitory posterior movement of the disc also occurs with the anterior mandibular repositioning. 


\section{References}

1- Ahmad M, Hollender L, Anderson Q, Kartha K, Ohrbach R, Truelove $E L$, et al. Research diagnostic criteria for temporomandibular disorders (RDC/TMD): development of image analysis criteria and examiner reliability for image analysis. Oral Surg Oral Med Oral Pathol Oral Radiol Endod. 2009; 107: 844-60.

2- Al-Ani Z, Gray RJ, Davies SJ, Sloan P, Glenny AM. Stabilization splint therapy for the treatment of temporomandibular myofascial pain: a systematic review. J Dent Educ. 2005; 69: 1242-50.

3- Badel T, Marotti M, Kern J, Laskarin M. A quantitative analysis of splint therapy of displaced temporomandibular joint disc. Ann Anat. 2009; 191:280-7.

4- Boero RP. The physiology of splint therapy: a literature review. Angle Orthod. 1989; 59: 165-80

5- Conti PC, Miranda JE, Conti AC, Pegoraro LF, Araújo CR. Partial time use of anterior repositioning splints in the management of TMJ pain and dysfunction: a one-year controlled study. J Appl Oral Sci. 2005; 13(4): 345-50.

6- Dao TT, Lavigne GJ . Oral splints: the crutches for temporomandibular disorders and bruxism? Crit Rev Oral Biol Med. 1998; 9: 345-61.

7- Demling A, Fauska K, Ismail F, Stiesch M. A comparison of change in condylar position in asymptomatic volunteers utilizing a stabilization and a pivot appliance. Cranio. 2009;27:54-61.

8- Drace JE, Enzmann DR. Defining the normal temporomandibular joint: closed-, partially open-, and open-mouth MR imaging of asymptomatic subjects. Radiology. 1990; 177:67-71.

9- Dworkin SF, LeResche L. Research diagnostic criteria for temporomandibular disorders: review, criteria, examinations and specifications, critique. J Craniomandib Disord. 1992;6:301-55.

10- Dylina TJ. A common-sense approach to splint therapy. J Prosthet Dent. 2001; 86: 539-45

11- Ekberg EC, Sabet ME, Petersson A, Nilner M. Occlusal appliance therapy in a short-term perspective in patients with temporomandibular disorders correlated to condyle position. Int J Prosthodont. 1998; 11:263-8.

12- Ettlin DA, Mang $\mathrm{H}$, Colombo V, Palla S, Gallo LM. Stereometric assessment of TMJ space variation by occlusal splints. J Dent Res. 2008; 87:877-81

13- Gateno J, Anderson PB, Xia JJ, Horng JC, Teichgraeber J F, Liebschner MA. A comparative assessment of mandibular condylar position in patients with anterior disc displacement of the temporomandibular joint. J Oral Maxillofac Surg. 2004;62:39-43.

14- Gökalp H, Turkkahraman H. Changes in position of the temporomandibular joint disc and condyle after disc repositioning appliance therapy: a functional examination and magnetic resonance imaging study. Angle Orthod. 2000; 70:400-8.

15- Hall HD. Intra-articular disc displacement Part II: Its significant role in temporomandibular joint pathology. J Oral Maxillofac Surg. 1995; 53: 1073-9

16- Hasegawa Y, Kakimoto N, Tomita S, Honda K, Tanaka Y, Yagi K, et al. Movement of the mandibular condyle and articular disc on placement of an occlusal splint. Oral Surg Oral Med Oral Pathol Oral Radiol Endod. $2011 ; 112: 640-7$
17- Jokstad A, Mo A, Krogstad BS. Clinical comparison between two different splint designs for temporomandibular disorder therapy. Acta Odontol Scand. 2005; 63:218-26.

18- Kurita H, Kurashina K, Baba H, Ohtsuka A, Kotani A, Kopp S. Evaluation of disk capture with a splint repositioning appliance: clinical and critical assessment with MR imaging. Oral Surg Oral Med Oral Pathol Oral Radiol Endod. 1998; 85:377-80.

19- Kurita H, Kurashina K, Ohtsuka A, Kotani A. Change of position of the temporomandibular joint disk with insertion of a disk-repositioning appliance. Oral Surg Oral Med Oral Pathol Oral Radiol Endod. 1998; 85: 142-5.

20- Kurita H, Ohtsuka A, Kobayashi H, Kurashina K. A study of the relationship between the position of the condylar head and displacement of the temporomandibular joint disk. Dentomaxillofac Radiol. 2001; 30: 162-5.

21- Kurita H, Uehara S, Yokochi M, Nakatsuka A, Kobayashi H, Kurashina K. A long-term follow-up study of radiographically evident degenerative changes in the temporomandibular joint with different conditions of disk displacement. Int J Oral Maxillofac Surg. 2006; 35(1):49-54.

22- Liu MQ, Chen HM, Yap AU, Fu KY. Condylar remodeling accompanying splint therapy: a cone-beam computerized tomography study of patients with temporomandibular joint disk displacement. Oral Surg Oral Med Oral Pathol Oral Radiol. 2012; 114:259-65.

23- Moloney F, Howard JA. Internal derangements of the temporomandibular joint. III. Anterior repositioning splint therapy. Aust Dent J. 1986; 31:30-9.

24- Okeson JP. Management of temporomandibular disorders and occlusion. $7^{\text {th }}$ ed. Saint Louis: Mosby; 2012

25- Santacatterina A, Paoli M, Peretta R, Bambace A, Beltrame A. A comparison between horizontal splint and repositioning splint in the treatment of "disc dislocation with reduction". Literature meta-analysis. J Oral Rehabil. 1998;25(2):81-8.

26- Simmons HC $3^{\text {rd }}$, Board of Directors, American Academy of Craniofacial Pain. Guidelines for anterior repositioning appliance therapy for the management of craniofacial pain and TMD. Cranio. 2005; 23: 300-5.

27- Tecco S, Festa F, Salini V, Epifania E, D'Attilio M. Treatment of joint pain and joint noises associated with a recent TMJ internal derangement: a comparison of an anterior repositioning splint, a fullarch maxillary stabilization splint, and an untreated control group. Cranio. 2004;22:209-19.

28- Wassell RW, Adams N, Kelly PJ. The treatment of temporomandibular disorders with stabilizing splints in general dental practice: one-year follow-up. J Am Dent Assoc. 2006; 137: 1089-98.

29- Whyte AM, McNamara D, Rosenberg I, Whyte AW. Magnetic resonance imaging in the evaluation of temporomandibular joint disc displacement - a review of 144 cases. Int J Oral Maxillofac Surg. 2006; 35:696-703. 